\title{
Estudo dA EficiênCIA CATAlítica do CÁlCIO E do Cobalto em Zeólita Beta na Decomposição de Óxido
} e-Xactal Nitroso na Presença de OXIGÊNIO OU VAPOr de ÁGUa

ISSN: 1984-3151

\author{
Study of Catalytic Efficiency of Calcium and Cobalt in Beta \\ ZeOLITE IN THE DeComposition OF NitRous OXIDE IN THE \\ Presence of Oxygen or Water Vapor
}

\author{
Nicole Moura Romeros Rabelo'; Marcelo da Silva Batista² \\ 1 Graduanda em Engenharia em Engenharia Química. \\ Universidade Federal de São João Del-Rei. Ouro Branco, \\ MG. nicole.moura.rr@hotmail.com . \\ 2 Doutor em Engenharia Química. UFSCar/2002. Professor \\ da UFSJ. Ouro Branco, MG. marcelobatista@ufsj.edu.br.
}

Recebido em: 19/03/2016 - Aprovado em: 20/09/2016 - Disponibilizado em: 30/11/2016

\begin{abstract}
RESUMO: Várias tecnologias estão sendo desenvolvidas para a redução das emissões dos óxidos de nitrogênio $\left(N O_{x}\right)$ e as técnicas mais eficientes envolvem o uso de catalisadores. Neste trabalho investigou-se a decomposição do óxido nitroso sobre Ca-beta, Co-beta e a zeólita na forma ácida (H-beta). Os catalisadores foram caracterizados por difratometria de raios $X(D R X)$ e por redução com hidrogênio a temperatura programada (TPR- $\mathrm{H}_{2}$ ). Os resultados mostraram a conservação da topologia da zeólita beta após o processo de preparação, ressaltando que não foram observados indícios de óxidos de cobalto ou óxidos de cálcio, e que os cátions estão em posição de intercâmbio. Os resultados mostraram que os catalisadores $\mathrm{H}$-beta e Ca-beta possuem baixa atividade na reação de decomposição do $\mathrm{N}_{2} \mathrm{O}$. No entanto, altas conversões foram observadas sobre Co-beta e a presença de $\mathrm{O}_{2}$ ou vapor de água não influenciou a atividade catalítica.

PALAVRAS-CHAVE: Poluição. Óxido nitroso. Catalisador. Zeólita beta.
\end{abstract}

ABSTRACT: Various technologies are being developed for reduction of nitrogen oxides emission $\left(N O_{x}\right)$ and the more efficient techniques involve the use of catalysts. This paper investigated the decomposition of nitrous oxide on Cabetha, Co-betha, and betha zeolite in acid form ( $H$-betha). The catalysts were characterized for $X$-ray diffraction (XRD) and temperature programmed reduction by $\mathrm{H}_{2}\left(\mathrm{H}_{2}\right.$-TPR). The results showed that the betha zeolite topology was maintained after the preparation process, revealing that cobalt oxides or calcium oxide have not been observed, and the cations are in interchange position. The results showed that $\mathrm{H}$-Beta and Ca-Beta catalysts have low activity in the $\mathrm{N}_{2} \mathrm{O}$ decomposition reaction. However, high conversions were observed on Co-Beta and the presence of $\mathrm{O}_{2}$ or water vapor had no effect on catalytic activity.

KEYWORDS: Pollution. Nitrous oxide. Catalyst. Betha zeolite.

\section{INTRODUÇÃo}

O aumento da taxa de substâncias nocivas na atmosfera está afetando o equilíbrio do meio ambiente e de suas formas de vida (MARCON, 2013). Esse fenômeno contribui para a acidificação de rios e florestas, o efeito estufa, o aumento de problemas 
respiratórios e circulatórios da população, o smog urbano e a depleção do ozônio estratosférico (DRUMM et al., 2014; LONGATI et al., 2014).

Dentre os poluentes atmosféricos, o óxido nitroso $\left(\mathrm{N}_{2} \mathrm{O}\right)$ contribui para a destruição da camada de ozônio, tem potencial de aquecimento 300 vezes maior do que $\mathrm{O}_{\mathrm{CO}_{2}}$ e, sua emissão anual é de aproximadamente 4,7 milhões t/ano (TOLMAN, 2010; NETO, 2014). Estima-se que a sua concentração atmosférica tenha aumentado em $9 \%$, comparada aos níveis pré-industriais, notando-se um crescimento anual na taxa de emissão de $0,2-0,3 \%$ causado pela intensificação de atividades antrópicas (PÉREZRAMíREZ et al., 2002; LIU et al., 2012).

Devido à baixa reatividade do $\mathrm{N}_{2} \mathrm{O}$, esse gás pode permanecer na atmosfera por cerca de 120 anos (NETO, 2014). Na tentativa de reduzir as emissões de $\mathrm{N}_{2} \mathrm{O}$, tecnologias envolvendo o uso de catalisadores estão entre as mais promissoras devido à decomposição de $\mathrm{N}_{2} \mathrm{O}$ em produtos inofensivos, como $\mathrm{N}_{2}$ e $\mathrm{O}_{2}$ (DAl et al., 2012).

$\mathrm{Na}$ busca por um catalisador ativo, seletivo, estável e de baixo custo para a decomposição de $\mathrm{N}_{2} \mathrm{O}$, se destacam os estudos usando zeólitas. Em particular, a zeólita beta possui características atraentes e vantajosas para a catálise, como ampla abertura de poros, grande área superficial e seletividade de forma (SILVA, 2008). Apresenta capacidade de gerar centros ácidos e trocar cátions de compensação, além de exibir alta estabilidade térmica e hidrotérmica (LONGATI et al., 2014; OLIVEIRA, 2002). Mesmos com essas características fascinantes, existem poucos trabalhos com a zeólita beta (LIU et al., 2012).

Zeólitas ZSM-5 e USY intercambiadas com íons de metais de transição são comumente utilizadas na redução das emissões de óxidos de nitrogênio. Os cátions de ferro, cobalto e cobre são geralmente os mais empregados, devido às satisfatórias atividades na decomposição dos óxidos de nitrogênio (BATISTA;
WALLAU; URQUIETA-GONZÁLEZ, 2005; SILVA, 2008). Além disso, esses metais são relativamente baratos, e economicamente viáveis. Entretanto, a atividade catalítica desses catalisadores, geralmente é reduzida na presença vapor de água e oxigênio, comumente presente nas emissões industriais (FERNANDES, 2005; SATSUMA et al., 2000).

O cobalto é amplamente utilizado na reação de decomposição dos óxidos de nitrogênio, na forma de óxidos suportado em alumina ou intercambiado na zeólita ZSM-5 (CRUZ; MASCARENHAS; ANDRADE, 1998; LUCAS et al., 2004; SILVA, 2008; SMEETS et al., 2008; WILCZKOWSKA et al., 2010). Li e Armor (1992) realizaram estudos de redução de $\mathrm{NO}_{x}$ com metano, na presença de oxigênio, sobre Co-ZSM-5, e os resultados mostraram que o catalisador manteve a atividade catalítica. No entanto, o metano precisa ser adicionado como reagente na mistura poluente e, se não for totalmente consumido, sua emissão na atmosfera contribui para o efeito estufa.

Estudos que empregam o cálcio são mais raros na literatura. Muramatsu et al. (1997) e SATSUMA et al. (2000) observaram que a redução de $\mathrm{NO}_{x}$ com metano não foi inibida pela adição de oxigênio sobre o catalisador de óxido de cálcio.

Nesse contexto, o objetivo deste trabalho foi investigar as características e o comportamento dos catalisadores Co-beta e Ca-beta na decomposição do óxido nitroso, na presença de oxigênio e vapor de água. O comportamento dos catalisadores Co-beta e Ca-beta, também foi comparado com a forma ácida da zeólita beta (H-beta).

\section{Metodologia}

Neste trabalho utilizou-se a zeólita $\mathrm{NH}_{4}$-beta comercial (TRICAT, $\mathrm{SiO}_{2} / \mathrm{Al}_{2} \mathrm{O}_{3}=24$ ) para a preparação dos catalisadores Ca-beta, Co-beta e H-beta. O catalisador H-beta foi preparado por calcinação da zeólita $\mathrm{NH}_{4}$-beta comercial em mufla, a $650^{\circ} \mathrm{C}$ por $2 \mathrm{~h}$. 
O catalisador Ca-beta foi preparado por três trocas iônicas consecutivas, a $50^{\circ} \mathrm{C}$, em placa de aquecimento com agitação magnética constante. Cada troca iônica teve duração mínima de 9h, e foi utilizado $1 \mathrm{~g}$ de zeólita para cada $50 \mathrm{~mL}$ de solução de 1 mol.L-1 de nitrato de cálcio $\left(\mathrm{Ca}\left(\mathrm{NO}_{3}\right)_{2} \cdot 4 \mathrm{H}_{2} \mathrm{O}\right.$, Isofar). Após o término de cada troca iônica, a suspensão foi filtrada a vácuo e colocada para secar em estufa. Após a terceira troca iônica a suspensão foi lavada com água destilada, e seca em estufa. Posteriormente, o catalisador foi calcinado em mufla a $650^{\circ} \mathrm{C}$ por $2 \mathrm{~h}$.

O catalisador Co-beta também foi preparado pelo mesmo procedimento usado para Ca-beta, utilizando, porém, solução de $1 \mathrm{~mol} . \mathrm{L}^{-1}$ de nitrato de cobalto II $\left(\mathrm{Co}\left(\mathrm{NO}_{3}\right)_{2} \cdot 6 \mathrm{H}_{2} \mathrm{O}\right.$, Êxodo Científica).

As análises de difratometria de raios $X(D R X)$ foram realizadas pelo método do pó, em um difratômetro Rigaku (Miniflex 600) com tubo de Cu e filtro de níquel operado com radiação CuKa, tensão de $40 \mathrm{kV}$, corrente de $15 \mathrm{~mA}$. A velocidade do goniômetro utilizada foi de $2^{\circ}(2 \theta) / \mathrm{min}$, com variação do ângulo de $5^{\circ}$ a $80^{\circ}(2 \theta)$. A partir dos dados obtidos, calculou-se o tamanho médio dos cristais usando a equação de Scherrer (Equação 1).

$$
\mathrm{D}_{\mathrm{h}, \mathrm{k}, \mathrm{l}}=\frac{(K \cdot \lambda)}{(\beta \cdot \cos \theta)}
$$

O tamanho do cristal foi obtido pela largura do pico mais intenso à meia altura $(\beta)$, usando comprimento de onda 0,1542 nm $(\lambda)$, e a dispersão esférica representada por 0,9 (K) (TAVARES, 2013).

As análises de redução com hidrogênio a temperatura programada $\left(T P R-H_{2}\right)$ foram realizadas em um equipamento SAMP3 (Termolab equipamentos Ltda.). Em cada análise, utilizaram-se $50 \mathrm{mg}$ de catalisador, que foram acondicionados com lã de quartzo em reator de quartzo tipo "U". Pela amostra passou um fluxo de $50 \mathrm{~mL} \cdot \mathrm{min}^{-1}$ da mistura gasosa composta por
$2 \% \mathrm{H}_{2} / \mathrm{Ar}(\mathrm{v} / \mathrm{v})$, enquanto a temperatura aumentou linearmente $\left(10^{\circ} \mathrm{C} \cdot \mathrm{min}^{-1}\right)$ da temperatura ambiente até $900^{\circ} \mathrm{C}$.

Nos ensaios catalíticos foram utilizados $50 \mathrm{mg}$ de catalisador em reator de quartzo tipo "U", alimentado com fluxo contínuo de $50 \mathrm{~mL}$. $\mathrm{min}^{-1}$ de uma mistura gasosa contendo $10 \%$ de $\mathrm{N}_{2} \mathrm{O}$ em hélio $(\mathrm{v} / \mathrm{v})$. A temperatura foi variada de $25^{\circ} \mathrm{C}$ até $600^{\circ} \mathrm{C}$, com intervalos de $50^{\circ} \mathrm{C}$ (em $5 \mathrm{~min}$ ). Para avaliar o comportamento catalítico na presença de $\mathrm{O}_{2}$ e $\mathrm{H}_{2} \mathrm{O}$, foram adicionados $10 \%$ de oxigênio ou vapor de água (via saturador) a $600^{\circ} \mathrm{C}$. O reator foi acoplado em linha a um espectrômetro de massas (THERMO) para análise de: $\mathrm{N}_{2}$ (28), $\mathrm{O}_{2}$ (32 e 16), $\mathrm{N}_{2} \mathrm{O}$ (44 e 30), $\mathrm{H}_{2} \mathrm{O}$ (18) e $\mathrm{He}$ (4). A conversão de $\mathrm{N}_{2} \mathrm{O}$ foi calculada através da Equação 2.

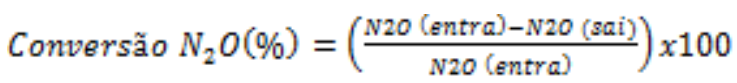

\section{Resultados e Discussão}

Os resultados da difratometria de raios $\mathrm{X}$ dos catalisadores $\mathrm{Ca}$, Co e H-beta estão apresentados na Figura 1. Ao comparar o difratograma da zeólita $\mathrm{H}$ beta com outros estudos, e com fichas cristalográficas do "X'Pert HighScore" (X'Pert cod.: 48-0074), identificaram-se picos próximos a $2 \Theta=7,7^{\circ}$ e $22,4^{\circ}$, que são característicos da zeólita beta (LOIHA, 2008; LONGATI et al., 2014; TAVARES; SILVA; BATISTA, 2014). Confirmou-se que a estrutura cristalina da $\mathrm{H}$ beta não sofreu alteração com o tratamento térmico e, a ausência de outras estruturas cristalinas na amostra. 


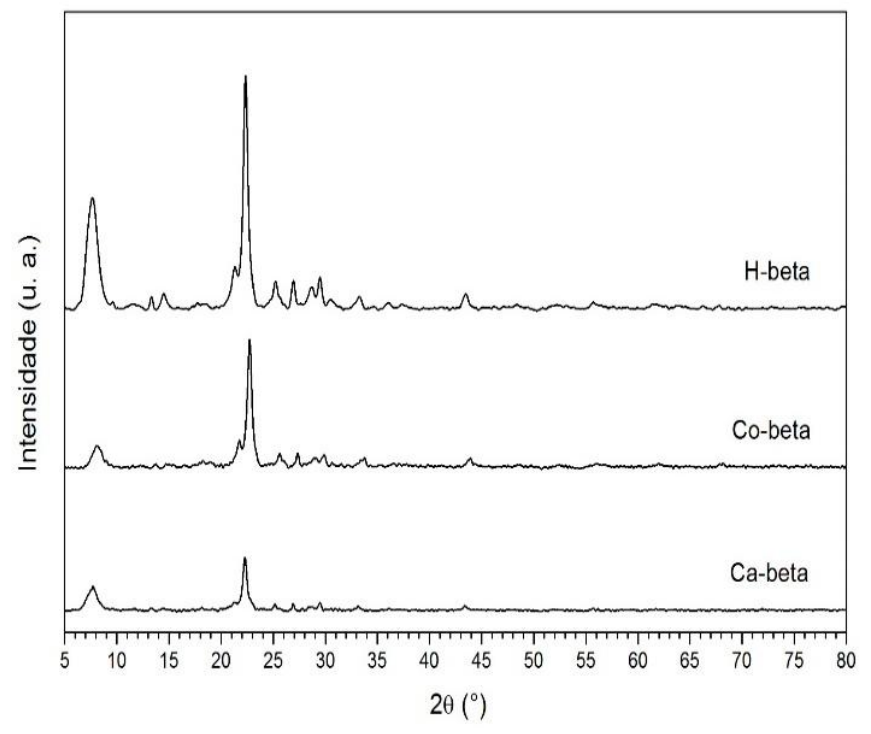

Figura 1 - DRX dos catalisadores ( $\mathrm{Ca}$, Co e H)-beta. Fonte - Próprio autor.

Os perfis de DRX dos catalisadores Ca-beta e Co-beta se assemelham ao difratograma da H-beta, apresentando os picos característicos da zeólita beta, porém, com menores intensidades. Isso indica que o processo de preparação e o cátion intercambiado na zeólita beta não modificaram sua topologia.

Não foram observados picos característicos de $\mathrm{CoO}$ ou $\mathrm{Co}_{3} \mathrm{O}_{4}$ no catalisador Co-beta, nem picos de $\mathrm{CaO}$ no catalisador Ca-beta. Pode-se afirmar então, que os cátions de cálcio e cobalto estão intercambiados e compensando carga na zeólita beta.

A Tabela 1 mostra o tamanho médio dos cristais dos catalisadores Ca-beta, Co-beta e H-beta, calculados pela equação de Scherrer. Observou-se que 0 tamanho médio do cristal está entre 19 e $20 \mathrm{~nm}$, indicando que os catalisadores são materiais nanocristalinos. Esses resultados estão de acordo com o estudo de Camblor, Corma e Valencia (1998), que obteve tamanhos de cristais entre $15-20 \mathrm{~nm}$ para a zeólita $\mathrm{H}$-beta $(\mathrm{Si} / \mathrm{Al}=12,5)$. O tamanho médio dos cristais permite observar que não houve aumento significativo do tamanho do cristal, inferindo-se que os óxidos de cobalto e cálcio não foram formados.
Tabela 1

Tamanho médio dos cristais de Ca-beta, Co-beta e Hbeta.

\begin{tabular}{c|c}
\hline Catalisador & Tamanho Cristal $(\mathrm{nm})$ \\
\hline Ca-beta & 19,29 \\
Co-beta & 19,82 \\
H-beta & 19,39 \\
\hline \multicolumn{2}{c}{ Fonte - Próprio autor. }
\end{tabular}

$\mathrm{Na}$ Figura 2 são apresentados os perfis de TPR- $\mathrm{H}_{2}$ dos catalisadores $\mathrm{Ca}$, Co e $\mathrm{H}$-beta. Observa-se que na faixa de temperatura estudada, os catalisadores $\mathrm{Ca}$ beta, Co-beta e H-beta não apresentaram picos de redução. As oscilações das curvas são atribuídas às pequenas variações da linha de base. Dessa forma, pode-se afirmar que os cátions de $\mathrm{Ca}^{2+}$ presentes em Ca-beta e, os cátions $\mathrm{Co}^{2+}$ presentes em Co-beta, não são facilmente redutíveis. Atribui-se tal comportamento ao ambiente de relativa estabilidade, formado por esses cátions em coordenação com os átomos de oxigênio da rede zeolítica. A redução pode ocorrer em temperaturas mais elevadas, pois é necessária maior energia de ativação (ZOLA, 2006).

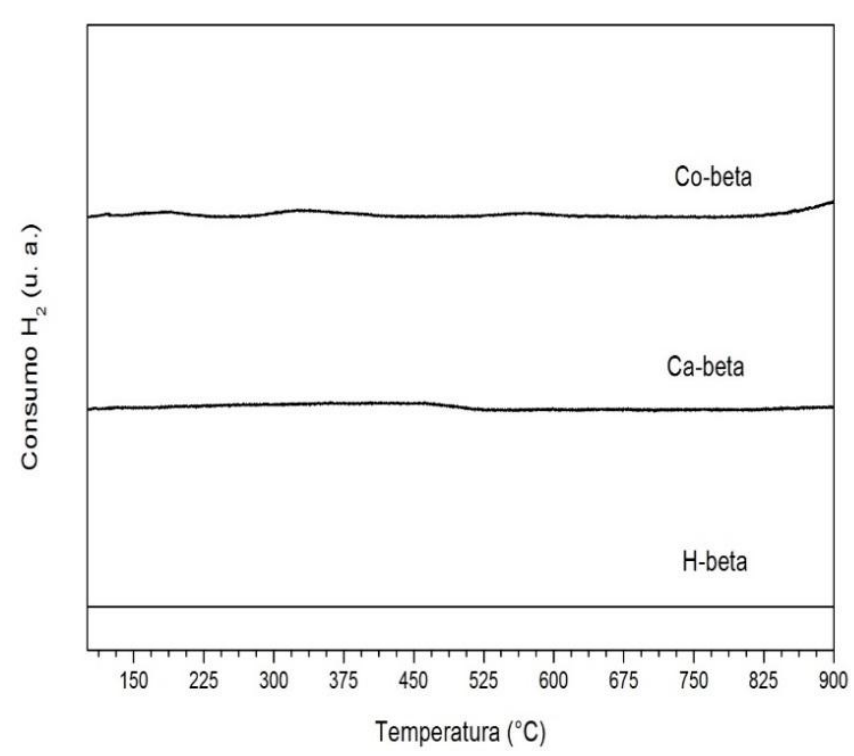

Figura 2 - TPR dos catalisadores (Ca, Co, e H)-beta. Fonte - Próprio autor. 
A atividade dos catalisadores foi avaliada na reação de decomposição do óxido nitroso, representada pela Equação 3.

$$
\mathrm{N}_{2} \mathrm{O} \rightarrow \mathrm{N}_{2}+\frac{1}{2} \mathrm{O}_{2}
$$

$\mathrm{Na}$ Figura 3 são apresentadas as conversões de $\mathrm{N}_{2} \mathrm{O}$ em função da temperatura sobre os catalisadores $\mathrm{Ca}$ beta, Co-beta e H-beta. O catalisador Ca-beta apresentou baixa atividade na reação de decomposição do $\mathrm{N}_{2} \mathrm{O}$ durante toda a rampa de temperatura. Nota-se um leve aumento na conversão com o aumento da temperatura, atingindo a conversão máxima de $18 \%$ a $600^{\circ} \mathrm{C}$. Comportamento semelhante foi observado com o catalisador $\mathrm{H}$-beta que também apresentou baixa atividade catalítica na reação. Notase um aumento perceptível da conversão no intervalo de $550^{\circ} \mathrm{C}$ a $600^{\circ} \mathrm{C}$, passando de $11 \%$ para $45 \%$.

A maior atividade na reação de decomposição foi observada sobre 0 catalisador Co-beta. Esse catalisador apresentou aumento significativo da conversão a partir de $400^{\circ} \mathrm{C}$, passando de $13 \%$ a $98 \%$ em $550^{\circ} \mathrm{C}$, e atingindo $99,8 \%$ a $600^{\circ} \mathrm{C}$. Essa maior atividade de Co-beta pode estar associada à variação do estado de oxidação de +2 para +3 do cobalto, permitindo a realização do ciclo redox, e isso não acontece com o cálcio nem com a zeólita ácida.

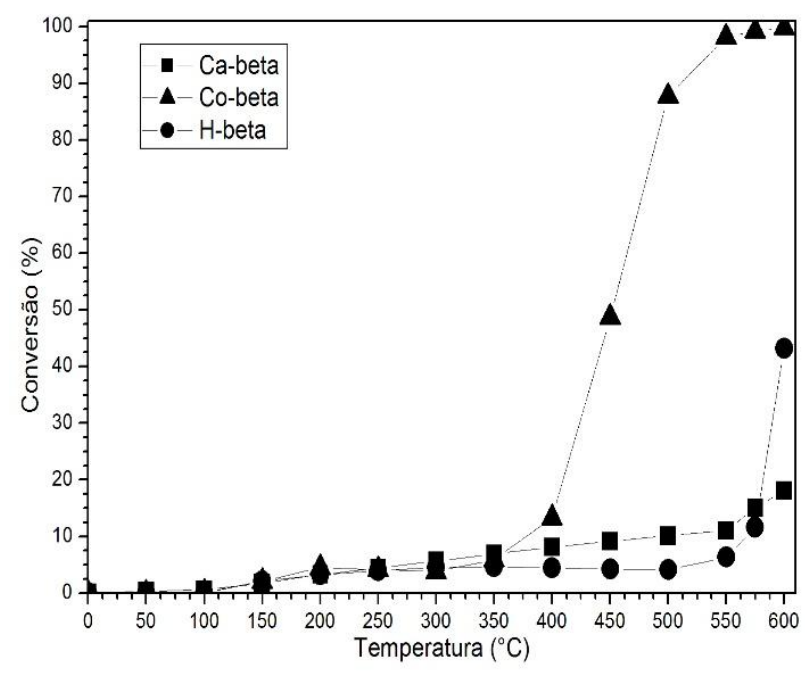

Figura 3 - Avaliação catalítica de (Ca, Co ou H)-beta na reação de decomposição do óxido nitroso.

Fonte - Próprio autor.
Os catalisadores Ca-beta e Co-beta foram avaliados na reação de decomposição do óxido nitroso, a $600^{\circ} \mathrm{C}$, na presença de oxigênio. Além da baixa atividade na decomposição de $\mathrm{N}_{2} \mathrm{O}$, o catalisador Ca-beta perdeu atividade na presença de oxigênio (resultado não mostrado). A Figura 4 apresenta as conversões do catalisador Co-beta na presença de oxigênio ou vapor de água, a $600^{\circ} \mathrm{C}$.

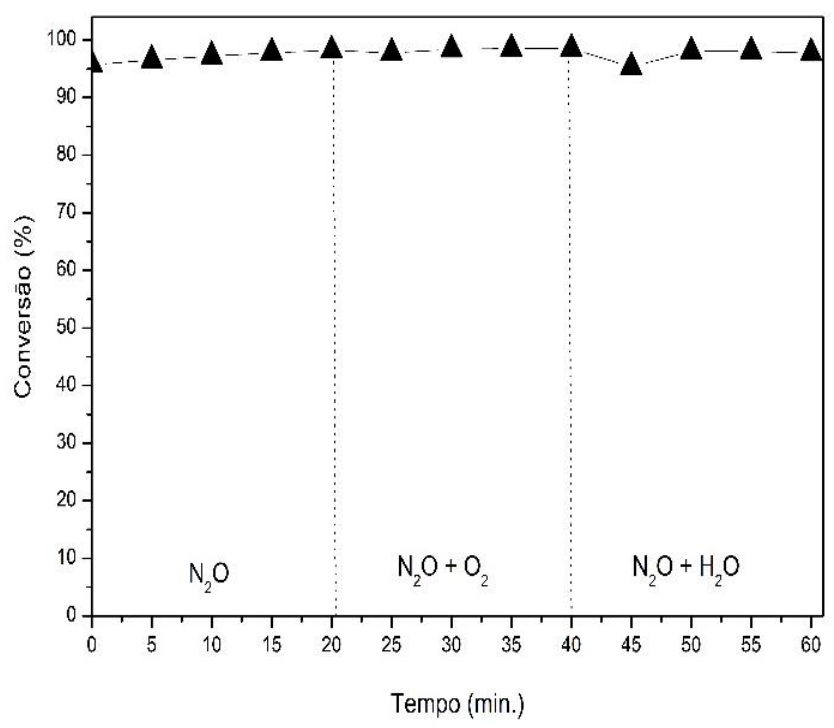

Figura 4 - Decomposição do óxido nitroso sobre Cobeta na presença de $\mathrm{O}_{2}$ ou vapor de água.

Fonte - Próprio autor.

O catalisador Co-beta manteve altas conversões de óxido nitroso, entre 95-98\%, na presença de oxigênio ou vapor de água. A presença de oxigênio ou vapor de água praticamente não influenciou a reação de decomposição do óxido nitroso sobre Co-beta, que continuou mostrando altas conversões de $\mathrm{N}_{2} \mathrm{O}$.

Os resultados mostram que Co-beta é um catalisador promissor para a decomposição do óxido nitroso. Ainda existem poucos estudos sobre o catalisador Cobeta e seu comportamento na presença de outros gases de exaustão, mas certamente estes resultados podem trazer novas perspectivas para decomposição do óxido nitroso. 


\section{CONCLUSÕES}

De acordo com os resultados obtidos, conclui-se que os catalisadores conservaram a topologia e estrutura cristalina da zeólita beta após o processo de preparação. Os óxidos de cálcio e cobalto não foram formados ou estão abaixo do limite de detecção do DRX. Dessa forma, afirma-se que os cátions cálcio ou cobalto estão intercambiados e, compensando carga na estrutura zeolítica.

As análises de TPR- $\mathrm{H}_{2}$ permitiram concluir que os cátions $\mathrm{Ca}^{2+}$ presentes no catalisador Ca-beta e, os cátions $\mathrm{Co}^{2+}$ presentes no catalisador Co-beta, não são redutíveis na faixa de temperatura estudada. O ambiente de relativa estabilidade formado por esses cátions em coordenação com os átomos de oxigênio da rede zeolítica, exigem maior energia de ativação para que a redução possa ocorrer.

A partir dos resultados da avaliação catalítica concluise que os catalisadores Ca-beta e $\mathrm{H}$-beta mostraram baixa atividade na reação de decomposição do óxido nitroso. O melhor desempenho catalítico foi observado para o catalisador Co-beta, que apresentou alta atividade catalítica em temperaturas superiores a $500^{\circ} \mathrm{C}$. A atividade de Co-beta na decomposição do óxido nitroso não foi influenciada pela presença de $\mathrm{O}_{2}$ ou vapor de água.

\section{AgradeCIMENTOS}

Os autores agradecem a CAPES/Branetec (projeto 012/2012) pelo apoio financeiro.

\section{REFERÊNCIAS}

BATISTA, M. S.; WALLAU, M.; URQUIETAGONZÁLEZ, E. A. Species active in the selective catalytic reduction of $\mathrm{NO}$ with iso-butane on ironexchanged ZSM-5 zeolites. Brazilian Journal of Chemical Engineering, v. 22, p. 341-351, 2005.

CAMBLOR, M. A.; CORMA, A.; VALENCIA S. Characterization of nanocrystalline zeolite Beta.

Microporous and Mesosporous Materials, v. 25, p.59-74, 1998.

CRUZ, R. S.; MASCARENHAS, A. J. S.; ANDRADE, H. M. C. Co-ZSM-5 catalysts for $\mathrm{N}_{2} \mathrm{O}$ decomposition. Applied Catalysis B: Environmental, v. 18, p. 223231, 1998.

DAI, C. et al. Reduction of $\mathrm{N}_{2} \mathrm{O}$ by $\mathrm{CO}$ over $\mathrm{Fe}$ - and Cu-BEA zeolites: An experimental and computational study of the mechanism. Microporus and

Mesoporous Materials, v. 167, p. 254-266, 2012.

DRUMM, F. C. et al. Poluição atmosférica proveniente da queima de combustíveis derivados do petróleo em veículos automotores. Revista Eletrônica em

Gestão, Educação e Tecnologia Digital, v. 18, p. 6678, 2014.

FERNANDES, J. E. Catalisadores Cu-, Co- ou FeZSM-5 - Caracterização e avaliação na redução de $\mathrm{NO}$ a $\mathrm{N}_{2}$ com hidrocarbonetos na presença ou ausência de vapor de água. Dissertação de Mestrado (Programa de Pós-Graduação em Engenharia Química) - Centro de Ciências Exatas e de Tecnologia, Universidade Federal de São Carlos, 2005.

LI, Y.; ARMOR, J. N. Catalytic reduction of nitrogen oxides with methane in the presence of excess oxygen. Applied Catalysis B: Environmental, vol.1, p.31-40, 1992.

LIU, N. et al. Comparative study on the direct decomposition of nitrous oxide over $\mathrm{M}(\mathrm{Fe}, \mathrm{Co}, \mathrm{Cu})$ BEA zeolites. Journal of Catalysis, vol. 294, p. 99112, 2012.

LOIHA, S. Synthesis of zeolite beta as a support of metal supported catalysts for toluene hydrogenation and application of in situ xas on catalysts characterization. Tese de Doutorado (Química) - School of Chemistry, University of Technology, 2008.

LONGATI, A. A. et al. Conversão Química de NO e CO sobre Catalisadores à Base de Óxidos de Cobalto ou de Ferro. Química Nova, v. 37, p. 221-225, 2014.

LUCAS, A. et al. Influence of the ion exchanged metal ( $\mathrm{Cu}, \mathrm{Co}, \mathrm{Ni}$ and $\mathrm{Mn}$ ) on the selective catalytic reduction of $\mathrm{NO}_{x}$ over mordenite and ZSM-5. Journal of Molecular Catalysis A: Chemical, vol. 225, p. 4758, 2004.

MURAMATSU, H. et al. Inhibitory effect of oxygen on catalytic removal of nitrous oxide with methane. In: International Symposium on Advanced Energy 
Conversion Systems and Related Technologies, 1997.

MARCON, J. B. Desenvolvimento de procedimentos para subsidiar as exigências legais de fiscalização e licenciamento ambiental com enfoque na poluição atmosférica. Monografia Universidade do Extremo Sul Catarinense (UNESC), 2013.

NETO, N. E. S. P. Catalisadores à base de CuO, $\mathrm{Fe}_{2} \mathrm{O}_{3}$ ou $\mathrm{CuO}-\mathrm{Fe}_{2} \mathrm{O}_{3}$ suportados sobre $\gamma-\mathrm{Al}_{2} \mathrm{O}_{3}$, $\mathrm{ZrO}_{2}$ ou $\mathrm{ZrO}_{2} / \gamma-\mathrm{Al}_{2} \mathrm{O}_{3}$ para a redução de $\mathrm{NO}$ com CO. Dissertação de Mestrado, PPGEQ, Universidade Federal de São Carlos, 2014.

OLIVEIRA, A. M. Decomposição de NO em cobre suportado sobre zeólitas. Dissertação de Mestrado (Programa de Pós-Graduação em Engenharia Química) - Escola de Engenharia, Universidade Federal do Rio Grande do Sul, 2002.

PÉREZ-RAMíREZ, J. et al. Formation and control of $\mathrm{N}_{2} \mathrm{O}$ in nitric acid production Where do we stand today? Applied Catalysis B: Environmental, vol. 44 p. 117-151, 2002.

SATSUMA, A. et al. Effects of methane and oxygen on decomposition of nitrous oxide over metal oxide catalysts. Catalysis Today, vol.63, p. 347-353, 2000.

SILVA, E. D. Catalisadores à base de $\mathrm{Cu}$, Co ou $\mathrm{Fe}$ trocados ou suportados em zeólita USY Avaliação na redução de NO com CO. Dissertação de Mestrado, PPGEQ, Universidade Federal de São Carlos, 2008.
SMEETS, P. J. et al. Co-ZSM-5 catalysts in the decomposition of $\mathrm{N}_{2} \mathrm{O}$ and the SCR of $\mathrm{NO}$ with $\mathrm{CH}_{4}$ : Influence of preparation method and cobalto loading. Applied Catalysis B: Environmental, vol.84, p. 505513, 2008.

TAVARES, E. C. G. Hidrodesoxigenação Catalítica de Fenol usando o Upgrade de Bio-óleo para Produção de Biocombustível. Dissertação de Mestrado (Programa de Pós-Graduação em Tecnologias para o Desenvolvimento Sustentável) Universidade federal de São João Del-Rei, 2013.

TAVARES, E. C. G.; SILVA, A. H. M.; BATISTA, M. S. Hidrodesoxigenação de fenol sobre catalisadores CoHBeta e CuHBeta visando upgrade de bio-óleo para obtenção de biocombustíveis. In: $4^{\circ}$ Congresso Internacional de Tecnologias para o Meio Ambiente, 2014.

TOLMAN, W. B. Binding and activation of $\mathrm{N}_{2} \mathrm{O}$ at transition metal Centers: Recent Mechanistic Insights. National Institutes of Health, vol. 49, p.1018-1024, 2010.

WILCZKOWSKA, E. et al. Direct nitrous oxide decomposition with a cobalt oxide catalyst. Applied Catalysis A: General, vol. 389, p. 165-172, 2010.

ZOLA, A. S. Preparação e avaliação de catalisadores de cobalto suportado em diferentes zeólitas para a síntese de Fischer-Tropsch. UEM. Dissertação de mestrado. Departamento de Engenharia Química, 2006. 